\title{
Laboratorios artísticos colaborativos. Espacios transfronterizos de producción cultural
}

\section{Collaborative artistic laboratories. Transfrontier spaces of cultural production}

\author{
Antonio Collados-Alcaide \\ Departamento de Escultura.Universidad de Granada. \\ colladosalcaide@ugr.es
}

Recibido: 6 de diciembre de 2013

Aprobado: 29 de abril de 2014

\begin{abstract}
Resumen
El emerger del paradigma del código abierto y las demandas de los movimientos sociales han permeabilizado los modos de organización de las instituciones culturales modernas. El artículo analiza el surgimiento de una nueva espacialidad crítica y colaborativa y cómo ésta está transformando los modos de investigación y producción cultural actual. Se centra en presentar el potencial instituyente de las nuevas formas de cooperación que se están ensayando en lo que se define como laboratorios artísticos colaborativos. Espacios de investigación y creación híbridos, fundamentados en estructuras cooperativas y en red, que hacen emerger un nuevo cuerpo socio-técnico que obliga a replantear las condiciones orgánicas tradicionales que han demarcado los escenarios de producción de conocimiento y práctica artística. Palabras clave: instituciones culturales, política cultural, co-laboratorio trabajo en red, arte colaborativo.
\end{abstract}

Collados-Alcaide, A. (2015): Laboratorios artísticos colaborativos. Espacios transfronterizos de producción cultural. Arte, Individuo y Sociedad, 27(1) 45-64

\begin{abstract}
The appearance of the open code paradigm and the demands of social movements have permeated the ways in which today's cultural institutions are organized. This article analyzes the birth of a new critical and cooperative spatiality and how it is transforming current modes of cultural research and production. It centers on the potential for establishing the new means of cooperation that are being tested in what are defined as collaborative artistic laboratories. These are hybrid spaces of research and creation based on networked and cooperative structures producing a new societal-technical body that forces us to reconsider the traditional organic conditions of the productive scenarios of knowledge and artistic practice.
\end{abstract}

Keywords: cultural institutions, cultural policy, co-laboratory, networking, collaborative art.

Sumario: 1. Introduction. Espacialidad crítica y nuevos procesos instituyentes, 2. Cruzadores de fronteras. Espacios híbridos en las instituciones de lo común, 3. Laboratorios tecno-políticos como espacios de colaboración, 4. El centro social 2.0 como dispositivo espacial deseante, 5. Laboratorios artísticos colaborativos. Organicidad y modos de trabajo en red, 6. Conclusiones. Referencias. 


\section{Introducción. Espacialidad crítica y nuevos procesos instituyentes}

Desde los años sesenta a la actualidad, han surgido diferentes modalidades de crítica dirigida por artistas y teóricos, agentes culturales en general, a las instituciones de su campo disciplinar. El objetivo principal de esta crítica era evidenciar las relaciones de poder e intereses que se dan dentro de ellas y como éstas determinan no sólo sus políticas y programas culturales, sino también las estructuras de organización y modos de relación que se entretejen dentro de ellas y de éstas hacia los públicos que forman su "exterior constituyente". La razón de esta crítica institucional se fundamentaba en el intento de trasladar las formas de democracia radical, que se conquistaban en algunos espacios públicos, al mismo interior de las instituciones culturales, dominio que, al igual que otras instituciones de la modernidad, estaba tradicionalmente protegido del disenso social y la negociación política. En las décadas actuales algo hemos avanzado en este sentido, y los procesos de autocrítica y transformación orgánica de algunas instituciones llamadas progresistas, evidencian la posibilidad de ensayar modos experimentales instituyentes que reinventen los marcos institucionales tradicionales.

El emerger del paradigma del código abierto -de la creación colectiva- y las demandas de los movimientos sociales, han permeabilizado los modos de organización de las instituciones culturales hasta incorporar metodologías provenientes de otras estructuras organizacionales del tercer sector como: la asamblea, el trabajo en red, la agencia, etc., pero estos procesos no pueden esconder la dificultad de esquivar o sustraerse a las lógicas neoliberales, y a sus efectos más perversos dentro del campo cultural: principalmente la reapropiación de la crítica como cualidad regeneradora del sistema y los usos interesados de la imaginación creativa y el trabajo flexible, deslocalizado y autónomo -modelo laboral surgido de los procesos históricos que superaron el sistema fordista- como forma de subordinación del sujeto (el productor cultural) a una lógica de producción inmaterial que promueve su propia precarización (Boltanski y Chiapello, 2002).

En este contexto, están surgiendo nuevos espacios y procesos instituyentes ya conscientes de la imposibilidad de constituirse en externalidad -un afuera- capaz de obviar las tensiones que generan los poderes hegemónicos. Lo que intentan estas nuevas formas instituyentes es contrapesar las provocaciones del capitalismo actual llevando a cabo experimentaciones complejas que ni olviden la necesaria y continua tarea de tener localizado al "adversario" (Chantal Mouffe, 2007), ni establecer posiciones claras a la hora de interactuar y negociar con él.

Lo que actualmente está tratando de definirse es la noción y el grado de "autonomía" que pueden tener estas prácticas instituyentes respecto al sistema de cosas -al Estado, a sus instituciones o a los mercados, por ejemplo-, sabiendo que esta autonomía se traduce en términos relacionales, es decir, en función de los modos de configurar la posición adversaria desde la que expresamos nuestra crítica. Esto supone, por tanto, que una de las tareas principales que deben acometer los proyectos instituyentes es plantear y establecer el lugar desde el que hablar, su posición y, desde ella, revertir la tendencia habitual de los movimientos y espacios que se auto-definen como autónomos de rechazar la posibilidad de cooperar con su supuesta alteridad. En este sentido, podemos entender por "autonomía" justo esa capacidad para disponer 
el orden de cosas, para decidir el lugar desde el que efectuar nuestras decisiones políticas.

Esta toma de posición o postura ha sido definida por Toni Negri como "diagonal política" (Petcou, Petrescu y Querrien, 2007, p. 291), es decir, como el modo o voluntad que pueden adoptar los sujetos para relacionarse con las formas de biopoder empleadas por las instituciones del Estado. Para Negri es inevitable estar atravesado por esta formas de poder, pero estar atrapados en ellas no impide que podamos mantener relaciones indirectas o demarcadas por posicionamientos oblicuos o de plena resistencia.

Del lado de esta "diagonalidad", el propio Negri reconoce el surgimiento, en las últimas décadas, de espacios y formas de experimentación social y cultural a modo de propuestas que pueden plantearse de alguna manera como contrapoderes. Estas experiencias manifiestan posicionamientos complejos y formas de estar diversas que reaccionan a las lógicas, también a las exclusiones, de las instituciones culturales del Estado: desde la más absoluta negación y distanciamiento, a formas de colaboración o alianza de distinta intensidad y escala, con las que tratar de desarrollar las fuerzas sociales y culturales locales.

En este sentido, surge una nueva "creatividad biopolítica" capaz de actuar a nivel social, cultural y político, dando forma a lo que Doina Petrescu llama "Public space of proximity" [espacios públicos de proximidad] (Petrescu, 2005, p. 57), una territorialidad emergente en forma de instituciones autogestionadas, cooperativas de producción, educación y vida colectiva, centros sociales de proximidad, y otros modos de organización, de carácter aún más informal, como encuentros y ocupaciones temporales del espacio (por ejemplo el fenómeno rave en la Inglaterra de principios de los noventa), plataformas participativas, equipos y redes de colaboración, etc. La misma Doina Petrescu, junto con Constantin Petcou, ambos miembros del atelier d'architecture autogérée de París, han conceptualizado estas formaciones en la metrópolis otorgándoles la capacidad de constituirse en "alterotopías" (Petcou y Petrescu, 2007, p. 322), lugares donde se generan cracks e "intersticios" en el orden de lo cotidiano, espacios porosos que concentran energías sociales y potencian encuentros con "el otro", un otro que es, por definición, distinto y contradictorio. Estas alterotopías se convierten en espacios críticos que cuestionan el orden de la vida, experimentando formas con las que "des-aprender los usos y costumbres al servicio del capitalismo y ensayar otros usos singulares, mediante la producción de una nueva subjetividad colectiva y espacial acorde con los deseos de esa otredad o alteridad involucrada. A través de este tejido de deseos cotidiano, estas prácticas micro-espaciales introducen otras temporalidades, otras dinámicas (más a largo plazo, azarosas, colectivas y a veces autogestionadas) que hacen de estos espacios, ámbitos sujetos a una continua transformación auto-poiética" (Ibidem, 2007, p. 323), es decir, que no cesan en la tarea de auto-reinventar sus estructuras y componentes.

El propio Negri ve en este continuo, una experimentación social de cualidades estéticas (Petcou, Petrescu y Querrien, 2007, p. 292), en tanto que estos espacios intersticiales conforman un campo de tensión entre lenguajes y culturas, un lugar donde se dirimen relaciones de poder entre sujetos e instituciones, entre fuerzas de dominación y resistencias que tratan de no ser asimiladas, comprometiendo 
la formación, estado y modos de relación de los regímenes de vida de los sujetos contemporáneos. Las alterotopías dan lugar a una nueva dinámica de fuerzas, donde las relaciones de poder se coordinan estratégicamente para dar finalidad, e incluso más potencia, a este devenir instituyente -creativo, heterogéneo y múltiple- que pone en cuestión todo ordenamiento y regulación exterior a ellas.

Este es el terreno para una nueva organización biopolítica, corporal e intelectual, que reaccione a la dominación capitalista constituyendo espacios y prácticas experimentales fundamentadas en la acumulación de saberes y energías colectivas con las que dar lugar a formas de organización híbridas donde lo común pueda ser subjetivado.

Un poder constituyente produce sujetos, pero estos sujetos deben juntarse. La producción de subjetividad no es un acto de innovación o el destello de un genio, es una acumulación, una sedimentación y, de cualquier manera, algo en permanente movimiento. Es la construcción del común mediante la constitución de colectividades (Ibidem, 2007, p. 291).

¿Pero a qué tipo de construcciones podríamos remitir para vislumbrar la posibilidad de un común que deviene instituyente? ¿qué estrategias de organización social conocemos que hayan ensayado formas de escapar a los modos de dominación instituyendo espacios políticamente radicales?

\section{Cruzadores de fronteras. Espacios híbridos en las instituciones de lo común}

Henry A. Giroux (1997) defiende, apoyándose en las teorías feministas, posmodernas y poscoloniales, la necesidad de entablar procesos de deconstrucción de los marcos monolíticos y de poder en los que se construyen las representaciones y discursos culturales dominantes. Este proceso, señala Giroux, pasa en cierta medida por criticar, perturbar y transformar las políticas culturales universalistas y homogéneas mediante posturas en resistencia. Es decir, debemos entender la manera por la que el poder privilegia e impone determinados discursos, y como relega otros a los márgenes, oscureciendo la diversidad, la multiplicidad y la diferencia. Mediante esta comprensión, surge el desafío por emprender nuevas estrategias culturales, educativas y sociales que generen rupturas y discontinuidades en las formas públicas y relaciones sociales estructuradas desde la dominación. A partir de ahí, tratar de establecer políticas basadas en el reconocimiento de la diferencia, en la oposición y el diálogo, esto es, en la generación de procesos, experiencias, también prácticas instituyentes, que reconozcan estar en una posición relativa, provisional, variable, experimental, movediza, cambiante...Aquí surge el llamamiento de Giroux a "educadores y trabajadores culturales para que se conviertan en cruzadores de fronteras, [agentes] comprometidos en un esfuerzo por crear esferas públicas alternativas" (Ibidem, 1997, p. 36).

En las últimas décadas hemos visto emerger una continua experimentación, respecto a las formas y modos de organización, que ha sido relevante para reinventar las formas de lucha social contemporánea. La constitución de redes de cooperación transnacionales, en relación al acceso a los media, la defensa de los migrantes, los 
movimientos contra el precariado, el derecho a la ciudad, ... han dado lugar a una nueva institucionalidad capaz de interpelar a las instituciones clásicas y reinventar las formas de colaboración social. Esta nueva institucionalidad ha sido denominada, dentro de las redes discursivas de los movimientos sociales, como: instituciones de lo común, en movimiento o instituciones monstruo (Universidad Nómada, 1998).

Se refieren a un conjunto de iniciativas que, sin ser aún numerosas, constituyen experimentos políticos avanzados -laboratorios en la metrópolis- que ponen a prueba dentro del campo de lo social, pero también en la arena cultural, la activación de dispositivos "anómalos" e "híbridos" que tratan de dar lugar a formas de producción y gestión de lo común radicalmente democráticas, es decir, que estén abiertas a una administración permanente por parte de una organicidad social comprometida y respetuosa con lo público. Dispositivos monstruosos o anómalos respecto a las lógicas neoliberales, y de naturaleza híbrida, ya que ponen "en red recursos e iniciativas de corte muy heterogéneo y contradictorio que mezclan recursos públicos y privados, relaciones institucionales y de movimiento, modelos de acción no institucionales e informales con formas de representación quizá formal o representativa... elementos que resultan a la postre cruciales para irrumpir en las esferas públicas estatalizadas y/o privatizadas, transformándolas" (Ibidem, 1998).

Con el objetivo de satisfacer y dotar de estabilidad al tejido de deseos e intereses, las instituciones de lo común, dan lugar a procesos de autoorganización y creación de redes entre sujetos múltiples. Crean laboratorios de encuentro, en los que la estabilidad deviene en apuesta estratégica con la que facilitar hibridaciones entre singularidades, recomponiendo y produciendo subjetivaciones diversas con las que tratar de superar la fragilidad que sufren habitualmente los proyectos colectivos.

Para ello experimentan tipologías de gestión cooperativa diversas, sin renunciar a establecer alianzas operativas con instituciones culturales, políticas o académicas con las que puedan experimentar las rupturas y pliegues productivos que han ensayado sobre sus formas de gobierno, en una época en la que estas instituciones tradicionales manifiestan ciertas crisis: de legitimidad, reconocimiento o de capacidad de enunciación.

En las relaciones que estas instituciones de lo común o monstruosas mantienen con otras instituciones públicas resuenan ecos de las distintas oleadas de crítica institucional de épocas anteriores. Aunque su posicionamiento está demarcado por criterios de autonomía o emancipación, su resonancia crítica ha tenido efectos medibles de afección entre ellas, por lo que ya no podemos hablar de posiciones dicotómicas, ni siquiera de un afuera versus a un adentro inquebrantable o plano, como los dos ejes donde se posicionan la "anomalía" y el Estado. La institución de lo común trata de superar a la institución disciplinar, pero no -como también nos dicen los compañeros de la Universidad Nómada (Gual y Salvini, 2010)- para dejarlas atrás, olvidar o negarlas, sino para ejercer una suerte de desbordamiento con el que poder componer otros sitios donde, a través de la cooperación social, pueda reinventarse las formas de producción y difusión de la cultura y el conocimiento.

¿Pero qué formas o tipologías podrían adoptar estas instituciones de lo común? 


\section{Laboratorios tecno-políticos como espacios de colaboración}

La metrópolis contemporánea está siendo atomizada por flujos y organismos que abren nuevos espacios de convivialidad. Territorios intersticiales de composición heterogénea que reúnen y cohesionan fragmentos dispersos, tal como haría la red digital, dando lugar a plataformas complejas donde las prácticas culturales se insertan, en colisión con otros cuerpos de saberes, para dar lugar a procesos participativos y colaborativos que devienen espacios de producción cultural y educativa autónoma.

Se trata de experiencias que inician proyectos de investigación sobre unos ejes de trabajo específicos, en la que una comunidad heterogénea y abierta de interesados, es convocada o se alía para diseñar y ensayar procesos (tentativas) encaminados a unos objetivos comunes por los que se sienten afectados. Antonio Lafuente (2008), ha definido esta dinámica como "laboratorio sin muros", concepto que aplica a la metodología y estructura seguida dentro de los encuentros mantenidos en Medialab Prado, denominados Laboratorio del procomún.

El concepto de "laboratorio sin muros", un espacio poroso, sin fronteras, en el que es difícil diferenciar entre cualquier dentro-fuera, coexiste con otras definiciones similares o paralelas dadas en diferentes medios y contextos. En el informe que la UNESCO hizo público en el año 2005, bajo el título Hacia las sociedades del conocimiento (VV.AA, 2005, p. 119-122), se propuso la pertinencia de considerar los avances tecnológicos (las posibilidades conectivas de Internet) como una oportunidad para crear redes y centros de investigación colectivos, en los que instituciones y agentes situados en lugares dispersos, pudieran llegar a integrarse y colaborar en proyectos complejos, e incluso, considerar la facilidad de las redes de comunicación para favorecer la integración de perfiles profesionales o saberes múltiples en la conformación de equipos interdisciplinares. Esta coordinación en nodos de investigación colaborativos es designada en el informe con el nombre de "co-laboratorio".

Al investigador informático William Wulf debemos una primera definición del término: un co-laboratorio es un emplazamiento "sin paredes" o sin muros, un punto de encuentro y espacio de colaboración entre académicos, investigadores, estudiantes y público en general interesado en la conformación de espacios de aprendizaje en red, flexibles y participativos (Machal-Cajigas, 2004).

Bien sea por un aprovechamiento eficaz de las tecnologías de la comunicación o mediante la generación de espacios de encuentro donde agentes diversos puedan generar y compartir conocimiento, el fundamento central de un co-laboratorio estriba en que cualquiera que esté interesado en un proceso de investigación o en un proyecto creativo, encuentre en este espacio "un lugar donde pueda aportar sus conocimientos, experiencias o puntos de vista, ya que lo que interesa es la construcción de mapas de conocimiento colectivo en permanente desarrollo" (Cobo y Pardo, 2007, p. 52).

Reinaldo Laddaga (2010) compara algunos de los nuevos modelos de investigación, surgidos en el ámbito de las ciencias de la vida, con el devenir colaborativo de un abanico amplio de prácticas artísticas. Para ello retoma los estudios de la profesora Helga Nowotny sobre la condición "transdisciplinar" de algunos métodos científicos, para componer una trayectoria comparativa entre dos líneas evolutivas paralelas: las de la investigación artística y científica. Según Nowotny en las últimas décadas ha 
surgido lo que denomina "modelo 2" de investigación -diferenciado de un "modelo 1"- según el cual, las experiencias de estudio se desarrollarían directamente en diálogo con el contexto en el que suceden los problemas que tratan de investigarse.

Nowotny (2006) destaca en The potential of the transdisciplinarity algunos atributos del nuevo modo de producción de conocimiento (modelo 2):

- El primer atributo del modelo 2 es el hecho de que la investigación contemporánea se está trasladando más allá del espacio tradicional del laboratorio, es decir, los problemas se formulan desde el principio en diálogo con un gran número de actores y atendiendo a sus perspectivas sobre el tema. La inclusión de la gente y el medio, en los modos de investigación, conlleva entender este modelo como una experiencia contextual.

- El segundo atributo que destaca Nowotny está relacionado con el hecho de que en el modelo 2, son múltiples actores los que participan en el proceso de investigación, intentando convocar una heterogeneidad de habilidades y conocimientos en el proceso de resolver un problema de partida. En este caso nota como se desdibujan las jerarquías organizativas y se abren las cadenas de mando. Cualquier resolución dependerá en este caso, de la organización de una estructura colaborativa laxa en la que los problemas sean trabajados a partir de procesos comunicativos abiertos.

- Por último, el tercer atributo del modelo 2 sería la transdisciplinariedad. La estructura de investigación generada en este caso es concebida como fórum, lo que aporta una perspectiva de investigación muy distinta a la tradicional parcelación disciplinar. En estos marcos de producción intelectual emergente, tratan de aplicarse líneas de investigación que surgen del contexto, del conjunto de conversatorios e integraciones generativas que en él se producen. Esta dinámica genera itinerarios productivos que conllevan desplazamientos o saltos creativos entre disciplinas. Nowotny señala el carácter "transgresivo" y co-evolutivo de la condición transdiciplinar del modelo 2 , al romper tanto los límites disciplinares como las fronteras estructurales institucionales.

Alineado en una trayectoria programática y metodológica similar, Laddaga cita la conceptualización que los investigadores y sociólogos Michel Callon, Pierre Lascoumes y Yannick Barthe hacen sobre lo que denominan como "foros híbridos" o "laboratorios al aire libre". Según los autores, se trata de espacios abiertos, donde se forman colectividades de investigación en la que los científicos o técnicos se alían o trabajan conjuntamente con ciudadanos, activistas sociales, técnicos, organizaciones del tercer sector, agentes culturales, etc., conformando grupos heterogéneos para dar respuesta o solución a una problemática (una "controversia socio-técnica") que sería difícil resolver sin movilizar los recursos y saberes de las comunidades afectadas (Callon, Lascoumes y Barthe, 2009, p. 18). De este modo, los foros híbridos no son solamente escenarios de trabajo donde se facilita la colaboración entre expertos de distintos niveles (científicos, técnicos, populares, etc.), sino que se conciben también como espacios en los que grupos en principio subalternos, o tradicionalmente con escaso índice de impacto y participación en los procesos de resolución de controversias o problemas sociales y culturales, son tenidos en cuenta. En este sentido 
estos laboratorios sociales y científicos instituirían nuevos marcos de representación política en el ámbito de la investigación y producción de conocimiento. Los laboratorios al aire libre no tratarían de anular, según Callon, Lascoumes y Barthe, el modelo de investigación y experimentación confinada, es decir, inserta y circunscrita únicamente a la labor de escrutinio y conclusiones que realiza la comunidad científica en sus centros de investigación, sino que intentarían aportar otras derivas territoriales, nuevos campos de acción por los que articular formas mixtas de experimentación a modo de estaciones colectivas de investigación entre expertos científicos y locales.

La existencia de espacios de investigación y creación híbridos, fundamentados en estructuras cooperativas y en red, hacen emerger un nuevo cuerpo socio-técnico que obliga a replantear las condiciones orgánicas y territoriales tradicionales que han demarcado los escenarios de producción de conocimiento. Aceptar, por ejemplo, el valor de las experiencias y tentativas científicas y culturales en las que se integran fragmentos sociales (agentes, colectivos e instituciones), nos lleva a considerar las muchas posibilidades de este campo, abierto y expansivo, para construir o alcanzar metas de mayor calado ecológico y de una capacidad transductiva realmente efectiva.

En este sentido, notamos una tendencia fuerte, entre agentes culturales y políticos a generar espacios y tiempos en los que ensayar marcos más abiertos -integradoresy cooperativos para la invención, la producción y distribución de conocimientos. En los últimos años notamos la emergencia de un sin fin de proyectos culturales que han tomado la forma del laboratorio social y cultural -laboratorios al aire libre o sin muros- para desarrollar estrategias alternativas y resistentes al dominio de la subjetividad y los cuerpos, por parte de las fuerzas y exigencias del capital neoliberal. Muchas de estas alternativas están conformando estructuras "para-institucionales", plataformas colaborativas que emergen como resistencias creativas con una fuerte componente pedagógica. Se trata de espacios donde grupos heterogéneos comparten saberes y fabrican nuevos, por lo que dan lugar a proyectos que podemos llamar de "auto-educación".

No se trata de espacios que pudiéramos identificar exclusivamente como infraestructuras físicas, sino más bien como dispositivos sociales, que pueden tener o no una formalidad concreta, pero que en cualquier caso, construyen territorios conceptuales formales e informales en los que surgen sinergias y modelos organizacionales heterogéneos, en los que desarrollar formas de colaboración cultural y política en contexto.

\section{El centro social 2.0 como dispositivo espacial deseante}

Quizás el paradigma que mayor influencia ha ejercido en cuanto a la transformación de los modos organizacionales y operacionales dentro del mundo globalizado haya sido el de "la red". Bajo este modelo hiperconectivo emergen modos de colaboración que se organizan a partir de establecer trayectorias políticas comunes. En el campo de la producción cultural crítica, estas sinergias han derivado en la realización de esfuerzos diversos para diseñar y ensayar prototipos insólitos, extravagantes incluso, que responden a las demandas y necesidades de las redes y movimientos sociales de generar nuevas experiencias, espacios o plataformas de aspiración instituyente. 
Aunque el funcionamiento de las redes organizadas nos haría pensar en la capacidad de una transformación institucional en movimiento y a escala global, son otras manifestaciones las que nos gustaría resaltar brevemente para ejemplificar estrategias o tipologías de lo que hemos presentado como "instituciones de lo común". Como ya hemos comentado, se trata de un cierto tipo de instituciones que, sin renunciar a pertenecer a redes transnacionales, desarrollan su trabajo fundamentalmente en lo local, entendido éste como un territorio donde se conjuga una afectividad cercana y deseante que hace posible la proliferación de formas de vida colectiva. En lo local ponen en escena formas de creación "blandas o suaves", que intentan ser próximas e integradoras, intentando no demarcar distancias entre identidades y culturas, a partir del uso de lenguajes sobre-codificados o hipertécnicos. Hablamos de instituciones que tratan de reafirmar sus anomalías como acto de resistencia, frente a la presión homogeneizadora que amenaza la riqueza y heterogeneidad propia de lo común, pues lo común, como apunta Ned Rossiter, "es lo que se construye a través de relaciones de diferencia, tensión y disputa” (Rossiter, 2011, p. 96), un organismo por tanto vivo, evolutivo y desafiante.

Frente a modelos con una organicidad pesada (un museo, centro de arte, una universidad, ...) las instituciones de lo común crean escenarios y estructuras frágiles, temporales, móviles, ... capaces de responder coherentemente a la condición movediza y mutante de las prácticas sociales y culturales micro-políticas pero intentando crear, a su vez, marcos dotados de cierta consistencia y estabilidad.

La espacialidad crítica y en movimiento de los nuevos centros sociales, o "centros sociales 2.0" o de segunda generación (Sansonetti, 2008, p. 13-19), como ejemplo más palpable quizás de las llamadas instituciones de lo común, nos permite comprender la emergencia de un nuevo devenir instituyente poscolonial y posindustrial, que reconecta con la herencia y presupuestos de las distintas fases de la crítica institucional para corporeizar contra-modelos orgánicos de carácter ciertamente creativo y radical. Los centros sociales de segunda generación contravendrían la molaridad rígida de las instituciones culturales y educativas del Estado, ya que por su esencia dispersa y micro-política fundan una suerte de organicidad molecular que podríamos subjetivar, apropiándonos del concepto propuesto por Deleuze y Guattari, como "máquinas deseantes":

La verdadera diferencia radica, por tanto, entre las máquinas molares por una parte, tanto si son sociales, técnicas u orgánicas, y las máquinas deseantes, que pertenecen al orden molecular, por otra parte. Eso son las máquinas deseantes: máquinas formativas, cuyos propios fallos son funcionales y cuyo funcionamiento es indiscernible de la formación; máquinas cronógenas confundidas con su propio montaje, que operan por ligazones no localizables y localizaciones dispersas y hacen intervenir procesos de temporalización, formaciones en fragmentos y piezas separadas, con plusvalía de código, $y$ donde el todo es él mismo producido al lado de las partes...máquinas propiamente hablando, porque proceden por cortes y fujos, ondas asociadas y partículas, flujos asociativos y objetos parciales, induciendo siempre a distancia conexiones transversales, conjunciones polívocas... (Deleuze y Guattari, 1985, p. 296). 
La institucionalidad deseante, la máquina molecular auto-regeneradora que quieren ser esos centros sociales 2.0 o de segunda generación, es concebida desde su posición dentro de la ciudad, como lugar intersticial, brecha abierta en la lógica neoliberal para operar con otras lógicas, aquellas que unen y cruzan: aprendizaje, trabajo, militancia y vida. Máquinas formativas, cuyos propios fallos son funcionales... espacios de creación colaborativa, de producción autónoma de saberes, nodos entremedias (inbetween), entrefronteras y transfronterizos, instituciones que asumen su fragilidad como potencia para perpetuarse en la incertidumbre de saberse experimento, de devenir, laboratorio en movimiento.

Con el devenir de los centros sociales de nueva generación surgen en las metrópolis contemporáneas unos dispositivos espaciales en los que se experimentan formas de sociabilidad que intentan estar al margen de los imperativos económicos neoliberales. Las lógicas de intercambio mercantiles, que afectan tanto al régimen de producción de tangibles como a la generación de modos relacionales entre personas, tratan de ser reemplazadas en ellos por políticas de afectos y cuidados, por dinámicas de intercambio no clientelares, dando lugar a espacios solidarios, en los que los saberes y capitales se colectivizan, donde se tratan de resolver problemáticas sociales globales (precariedad laboral, derecho a la vivienda, urbanismo agresivo, crisis medio-ambientales, acceso a los media, control de la información, derechos de los migrantes, etc.) a partir de un trabajo contextual y localizado en una territorialidad concreta, barrios o ciudades, sin olvidar que esta escala de intervención 1:1 no limita la posibilidad de alcances mayores. El ejercicio de experimentación social, política y cultural que se da en cada centro social se multiplica o repliega al contacto con otras experiencias y procesos generadas en el conjunto de nodos de la red transnacional de colaboración y comunicación en la que suelen estar inscritos estos espacios. Las condiciones de reproducción de problemáticas globales en entornos locales nos lleva a concebir la espacialidad productiva de estos centros sociales en términos de laboratorio, en tanto que concentran muestras de problemáticas complejas sobre las que se intentan testear soluciones, abriendo los códigos estructurales que las componen. Para ello, en estos centros se realizan ejercicios de creatividad tecnopolítica que son llevados a cabo gracias a la acumulación de inteligencia colectiva.

La intensidad y variedad de usos, la naturaleza modular y recombinante de los procesos y experiencias que en ellos acontecen, así como las oficinas tácticas instaladas en su sitio hacen de ellos estaciones multidimensionales en las que se desarrollan formas de colaboración política y cultural que trascienden tanto los modelos de organización de las instituciones sociales y culturales modernas, como las formas de producción paradigmáticas y tradicionales en estos ámbitos.

Estos espacios actúan como nodos en los que de manera autónoma se juega y ensaya, se comparten conocimientos y se aprende, se erran procesos y se aciertan soluciones, constituyéndose como laboratorios sociales colaborativos. Como ha definido Marina Garcés -al notar esta emergencia en el contexto estatal- se trata de lugares donde el "aprender, enseñar, pensar, escribir y crear puedan exponerse a lo imprevisto, a lo desconocido, a la zozobra, a la experimentación que no se protege bajo resultados ya preestablecidos. En definitiva: espacios donde abrir preguntas que realmente importen y compartir saberes que verdaderamente nos afecten" (Garcés, 2010). 
El desarrollo histórico de los centros sociales ha ido a la par de las luchas por la defensa del procomún cultural, es decir, de aquellos esfuerzos realizados para contrarrestar la privatización y el control restrictivo del patrimonio cultural y el saber generado en colectividad. En esta tarea, las nuevas herramientas de comunicación tecnológica han ayudado y hecho posible no sólo la conexión entre individuos, con la consiguiente multiplicación de la capacidad de acceso y dispersión de información y conocimientos, sino que se den formas de producción cultural, organizadas en comunidades de interés más o menos estables, de mayor alcance y eficacia.

Por ejemplo, el desarrollo de la cultura libre, ha promocionado la creación de nuevos escenarios productivos en los que, desde el amateurismo o el do it yourself (hazlo tu mismo), se han generado plataformas colaborativas para la construcción de conocimientos aplicados o soluciones técnicas con las que abrir las "cajas negras" (Latour, 1992) de los medios y de otros mecanismos tecnológicos y sociales.

Vinculados a los centros sociales de nueva generación han surgido otros espacios de experimentación, en forma de laboratorios tecnopolíticos, que han tenido fuerte influencia en los modos de diseñar estructuras colaborativas para la creación y la lucha política: los hacklabs o los hackmeetings.

Los hacklabs encarnan el intento por situar las políticas relacionales y cooperativas dadas en la virtualidad de la red en un espacio local, físico, acoplando por tanto las cualidades que aporta la red con los beneficios que el trabajo especulativo, cuerpo a cuerpo, puede aportar. En ellos se crean redes de intercambio de saberes a partir de la colectivización de habilidades y de la pasión puesta en la experimentación con tecnologías que se quieren socializar. En los hacklabs se construye lo que Xabier Barandiaran define como un "espacio rizomático de saberes, técnicas e intervenciones teconopolíticas" (Barandiaran, 2006, p. 146), lo que supone un territorio donde se conjugan subjetividades diversas, agentes con distintos niveles de expertizaje técnico y lingüístico, conexiones entre personas, grupos y espacios que emprenden procesos de discusión, investigación y práctica con el objetivo de indagar y proponer modos de comprender el funcionamiento complejo de los mecanismos y sistemas tecnológicos.

Aunque en la mayoría de los centros sociales autogestionados han surgido estos espacios de experimentación medial, en muchos casos la precariedad e inestabilidad a la que están sujetos estos lugares ha dado lugar a fórmulas más flexibles para la convocatoria y construcción de estos laboratorios de experimentación cultural y técnica. Por ejemplo, los hackmeetings o algunas clases de laboratorios de medios móviles pueden ser algunas de las propuestas ideadas para expandir a la sociedad los modos de hacer de mediactivistas y otros agentes culturales interesados en la experimentación con las nuevas tecnologías de la información y comunicación. En ambas se intentan generar experiencias abiertas, en las que surjan oportunidades para rearticular los saberes expertos técnicos con otros cuerpos de saberes locales o populares, mediante la creación de sinergias entre participantes ocasionales, colaboradores asiduos u organizadores.

Una experiencia de interés para ejemplificar propuestas de investigacióncreación basadas en la generación de infraestructuras móviles aplicadas a replicar condiciones de laboratorio puede ser Makrolab, un proyecto ideado en el año 1994 por el artista esloveno Marko Peljhan. El signo más reconocible del proyecto es su 
unidad móvil de investigación, concebida por Peljhan como un laboratorio vivo, un espacio habitacional y de trabajo, desmontable y portátil, que permite reproducir unas condiciones de trabajo idóneas para un número reducido de personas incluso en condiciones de climatología extrema. Se trata de una estación equipada para alojar a artistas, científicos, trabajadores de medios tácticos, etc., cohesionando equipos mixtos para generar procesos de investigación sobre una serie de temáticas concretas, establecidas a modo de ejes de investigación del proyecto: el campo de las comunicaciones, los sistemas climáticos o los movimientos migratorios humanos, por ejemplo. Makrolab es un proyecto colaborativo, una experiencia de investigación sobre condiciones contextuales y sobre la propia experiencia cooperativa al interior de la estructura. Los resultados y documentaciones surgidas en los periodos de activación de la unidad, tratan de hacerse públicos a través de la red o de eventos culturales y científicos con la intención de generar un diálogo entre las experiencias promocionadas desde la unidad y otras investigaciones o procesos llevados a cabo desde el ámbito artístico o científico.

El laboratorio híbrido creado por Marko Peljhan opera en un campo abierto. Trata de romper barreras disciplinares y sociales. Su capacidad para mutar e itinerar conlleva entenderlo como un espacio de experimentación transfronteriza, un dispositivo que trata de transgredir las formas y medios de dominación contemporáneas, deconstruyendo los marcos rígidos de producción de saber, abriendo los códigos y lenguajes técnicos y generando políticas colaborativas entre agentes diversos. Makrolab por otra parte tiene una cualidad ecológica, es decir, se inserta y trabaja sobre contextos determinados. Estudia las condiciones y tensiones sociales, ambientales y comunicacionales de los lugares donde se sitúa para revelar información invisibilizada o generar modos en los que la complejidad informacional pueda ser legible (Holmes, 2007).

Una segunda experiencia que me gustaría reseñar, en este caso desarrollada en el contexto geopolítico del Estrecho de Gibraltar, es el encuentro Fadaiat. Celebrado en los años 2004 y 2005 entre las ciudades de Tarifa y Tanger y posteriormente en Barcelona y Málaga, Fadaiat consistió en la creación de un prototipo espacial, a la vez que evento artístico, tecnológico y social, muy en la tradición de los encuentros hackers (hackmeetings) o los hacking the streets, eventos en los que -como ya hemos comentado- tratan de romperse los muros de los laboratorios hackers (hacklabs), para sacar los saberes y comunicarlos a la calle, demostrando el potencial de uso social que poseen.

Fadaiat, que significa "por los espacios" en árabe culto, o "antena parabólica" y "nave espacial" en el uso cotidiano, fue definido por sus organizadores como un "laboratorio sobre trabajo, política y arte en la frontera" (Pérez de Lama, Soto, Moreno, 2003, p. 70). Fadaiat supuso una experiencia pionera de colaboración entre agentes tecno-activistas, artistas, arquitectos, activistas pertenecientes a diversos movimientos sociales y ciudadanos de ambos lados de la frontera marroquí-española. Un encuentro que funcionó como laboratorio de experimentación y debate entre diversas redes y sujetos "cuyo pensamiento y práctica artística y social se encontraba atravesada de diversas formas por los nuevos reclamos de libertad de movimiento y libertad de conocimiento" (VV.AA, 2006, p. 1). 
Durante varias jornadas de trabajo, en los encuentros Fadaiat se sucedían conferencias, seminarios, talleres y diversas actividades lúdicas, dirigidas a explorar nuevas formas de cooperación entre activistas de los derechos sociales, investigadores y agentes culturales para dar lugar a una plataforma de comunicación y trabajo colaborativo que pudiera ejercer de contrapoder comunicativo y táctico a los procesos de control y tergiversación informativa que dominaba y domina la frontera del Estrecho de Gibraltar. En las distintas convocatorias de Fadaiat se consiguió reunir un grupo heterogéneo de personas y colectivos, de diferente procedencia (nacional e internacional), estructura y objetivos programáticos (grupos de investigación sobre el territorio, representantes de casa sociales, colectivos mediactivistas, asociaciones culturales, artistas y urbanistas interesados en los flujos migratorios y de información, etc.), dando lugar a una de las primeras redes de trabajo nacionales en las que aparecía vinculada la práctica artística, la acción contra-informativa y la lucha social.

Desde aproximaciones diversas, las dos prácticas anteriores llevan a cabo experimentaciones en el campo social y cultural desde una perspectiva política radical. Generan espacios donde se ensayan e incentivan tentativas constitutivas de una nueva relacionalidad política. Frente a la hiper-regulación y exceso de control que imponen los órdenes sociales, políticos y económicos dominantes, la espacialidad difusa, móvil y mutante que entrevemos en los hacklabs, en unidades como Makrolab o en el acontecer tecnopolítico de Fadaiat, trata de rearticularse en redes relacionales que tienen como cualidad paradigmática el ser "cruzadoras de fronteras", tal y como hemos visto reclamar anteriormente a Giroux.

\section{Laboratorios artísticos colaborativos. Organicidad y modos de trabajo en red}

Finalmente, presentaremos algunos casos en los que trataremos de ejemplificar las cualidades de la espacialidad crítica que hemos denominado como "laboratorios artísticos colaborativos" y sus modos de hacer más significativos, intentando con ello plantear alternativas a la organicidad y estructura de nuestras instituciones y espacios culturales tradicionales.

Uno de los elementos a destacar, en la configuración orgánica de los laboratorios artísticos colaborativos, es la atención que muestran por el ensayo de protocolos de participación que transciendan las estructuras tradicionales de inclusión de públicos y agentes colaboradores. En este contexto el artista, colectivo o agente cultural ha de considerarse como un "facilitador" (Remesar, 2005), es decir, como un catalizador de encuentros, relaciones y procesos no predeterminados. Éstos, crearían las condiciones, o desarrollarían los mecanismos, por los que comunidades específicas o heterogéneas podrían sumarse y participar de la generación de procesos y espacios de colaboración, producción e intercambio de saberes, dando lugar a plataformas (de formalidad diversa) en las que desarrollar procesos significativos de aprendizaje y experimentación en red.

Un ejemplo de esto es The Public School, una experiencia liderada por la organización intermedial (arte, media y arquitectura), sin ánimo de lucro, Telic Arts Exchange. The Public School surge en el año 2007 en Los Ángeles, bajo la perspectiva de construir "una escuela sin curriculum" (The Public School), una plataforma que funciona a veces en espacios físicos concretos, aunque su línea habitual de producción 
está más encaminada a la generación de redes de intercambio inmateriales en las que entrecruzar conocimientos, habilidades y deseos.

Los inicios de The Public School están vinculados en un cambio en la perspectiva que la organización realizó sobre el programa de actividades que desarrollaban en su local. Situado en una zona de galerías del Chinatown de Los Ángeles, decidieron, tras cinco años aplicando un programa de exposiciones mensuales, modificar los objetivos con los que se abría su espacio para transformarlo de un lugar de presentación de proyectos a un lugar de producción y co-educación. En cierta medida, este cambio estaba vinculado a las necesidades detectadas por los miembros de Telic Arts Exchange en la comunidad artística local, necesitada de espacios de fricción e intercambio, y por su propio interés en proyectos de carácter procesual, transdisciplinares, contextuales y basados en protocolos de investigación (Simonson, 2009).

Este cambio de paradigma conllevó pensar una línea de funcionamiento estratégica por la que poder abrir el espacio a las propuestas e intereses de una comunidad mayor. El diseño de actividades no debería ya circunscribirse a la planificación hecha únicamente desde la organización, sino que necesitarían desarrollar mecanismos por los que atraer y vincular a más gente. La sede de Telic Arts Exchange debía conformarse en un lugar donde acontecieran agenciamientos diversos, no premeditados, e incluso imprevisibles, fruto de idear una estructura de participación ágil, inclusiva y atrayente. Si utilizáramos el lenguaje informático como metáfora, podríamos decir que The Public School trabajaría bajo una espacialidad y estructura entendida entonces en términos de código abierto. El funcionamiento de The Public School es sencillo: mediante un plataforma web habilitada a modo de boletín y buzón de ideas, se recopilan ofertas y demandas de actividades formativas. Una vez compiladas en la página web del proyecto y comentadas por los usuarios, son evaluadas por un comité asesor, cuya función es adecuar y equilibrar las ofertas y demandas, dando prioridad a aquellas actividades que según su criterio tienen mayor interés o un seguimiento más amplio. Si las propuestas son aceptadas, pasan, en colaboración con los demandantes u ofertantes, a redefinirse, gestionarse e incluir en programa. Para facilitar el seguimiento de las actividades, la web de The Public School funciona como calendario informativo y como plataforma para la gestión de las actividades.

La diversidad de ámbitos por los que se desplazan las actividades que se suceden en The Public School, conforman la extensión del proyecto, desde un punto de vista conceptual, pero también social, ya que la red de agentes vinculados a esta escuela experimental se expande en función de la naturaleza e identidad de las colaboraciones que se dan en ella.

La economía de intercambios de The Public School, tratar de abrir un espacio intersticial en el régimen de producción habitual del ámbito artístico. Cercano a las políticas del trueque, proyectos de esta naturaleza señalan una disrupción en las lógicas mercantilistas que guían cualquier sistema productivo inserto en los modos de relación tardocapitalistas.

La naturaleza medial de estos espacios de intercambio, hace que una de las mayores complejidades que puedan encontrarse para su funcionamiento y desarrollo, sea la dependencia al flujo de operaciones que la comunidad de participantes pueda generar, 
pero en ello está también su potencialidad. En este sentido, se trata de espacios de intensidad variable y con una evolución muy difícil de prever o aventurar. El reto de estos laboratorios está en la capacidad que tengan para abrir su código organizativo, acción que posibilitaría facilitar la participación en la estructura productiva del proyecto y, así, una verdadera rearticulación contextual o ecosistémica de la práctica.

La generación de protocolos, herramientas, recursos, etc. se ha constituido en una de las líneas de trabajo de mayor tensión e interés de las prácticas artísticas colaborativas, como bien ejemplifican los proyectos ya comentados. En cierta medida, esta profusión de plataformas de generación e intercambio de recursos está provocada por las propias deficiencias o limitaciones que muchas de las prácticas colaborativas encuentran al estar situadas, por convicción ética o por sufrir cierta marginalidad, en posiciones de producción y gestión autónoma tanto de recursos materiales como inmateriales. El desarrollo de las tecnologías de la comunicación, y la mayor facilidad y acceso a medios de producción y comunicación en red, ha contribuido a la emergencia de proyectos en red, en los que la generación de archivos y bancos de recursos, ha supuesto la construcción de nuevos espacios discursivos y políticos dispuestos a modo de contra-poderes de las hiper-normativas instituciones del saber y la cultura.

Por último nos gustaría introducir el trabajo del Stockyard Institute de Chicago, una plataforma para el desarrollo de proyectos que entrecruzan, entre otros ámbitos, la educación, el arte y el trabajo comunitario. En los últimos años, son muchos los grupos y colectivos artísticos y culturales que han enfatizado dentro de los objetivos de su práctica el desarrollado de herramientas específicas y elementos basados en la construcción de centros de recursos para artistas y educadores. En sus casi veinte años de actividad, el Stockyard Institute ha desarrollado proyectos diversos como: programas de investigación y acción sobre la ciudad, dispositivos de comunicación comunitaria, residencias y eventos artístico-educativos, ediciones de fanzines manuales y publicaciones, exposiciones, etc. Entre otros recursos, el Stockyard Institute ha desarrollado SITE, una plataforma colaborativa y fórum online con el que producir y compartir recursos educativos (impresos, audio y vídeo) que poder implementar en experiencias artísticas y de educación comunitaria.

El Stockyard Institute ha generado también algunos ejemplos paradigmáticos para entender cómo pueden ser articulados los espacios o eventos expositivos dentro de instituciones culturales como laboratorios artísticos y pedagógicos colaborativos. El caso quizás más reseñable sea Pedagogical Factory un evento múltiple y heterogéneo, celebrado en el Hyde Park Art Center de Chicago en el año 2007 y coordinado junto con la red cultural y ciudadana AREA, organizado con el objetivo de explorar y presentar alternativas al trabajo tradicional en educación y arte. Pedagogical Factory se instituyó en un "laboratorio temporal y abierto" (Stoyckard Institute, 2007) que consiguió articular una red de agentes, colectivos e instituciones que, de manera autónoma, trabajaran en la intersección entre el arte y la pedagogía en el espacio público.

Los objetivos concretos, expresados por los organizadores eran los siguientes:

- Desarrollar colaborativamente un texto inicial y un curriculum sobre estrategias artísticas contemporáneas para escuelas y espacios educativos alternativos. 
- Facilitar el acceso público a propuestas y estrategias para experimentar y crear una ciudad educada. El Hyde Park Art Center se habilitará como espacio de trabajo, encuentro, organización y producción...

Los grupos e iniciativas involucradas explorarán de forma crítica algunos de los siguientes temas:

- Las fábricas de hoy: ¿Cómo están afectando hoy los cambios económicos a la naturaleza del trabajo y la educación? ¿Son las ciudades y las escuelas las nuevas fábricas?.

- ¿Cómo hemos aprendido?: Explorando las tradiciones de la educación crítica y radical.

- Estrategias para una ciudad educada: Un examen de las herramientas, los experimentos, métodos y enfoques de aprendizaje, el conocimiento cívico y la acción colectiva (Hyde Park Art Center, 2007).

Para ello, el Hyde Park Center se transformó completamente, virando sus espacios expositivos a lugares de discusión y trabajo. Se instalaron dispositivos espaciales singulares que ayudaran a cualificar el espacio y hacerlo más adecuado para la experiencia colectiva que se iba a desarrollar. Una estación de radio por internet, un centro de investigación portátil, una librería especializada en arte, educación y movimientos sociales, un espacio para conferencias y seminarios, paneles expositivos, pizarras... todos estos dispositivos convirtieron este evento en un hito que marcó de una manera significativa el devenir de las redes artísticas y educativas de carácter crítico, incluso activistas, en la ciudad de Chicago. Del evento se obtuvieron algunos resultados palpables, como la realización de manuales y libros de texto sobre enseñanza artística para la escuelas secundarias. AREA, una red que aúna a artistas, investigadores, educadores y activistas de la ciudad, produjo un número de su revista semestral centrándolo en los debates sobre los elementos pedagógicos que se habían discutido durante el evento. Pedagogical Factory, no fue únicamente un lugar donde cuestionar y proponer cambios en los sistemas educativos tradicionales, sino que intento no caer en el discurso retórico inmovilista, convirtiéndose en un laboratorio alternativo, en el que experimentar las formas de colaboración y co-aprendizaje que estaba propugnando.

\section{Conclusiones}

Las prácticas artísticas colaborativas desarrollan en su seno procesos colectivos de generación de conocimientos, es decir, metodologías de trabajo en las que los diferentes sujetos que intervienen ponen a disposición el conjunto de saberes que atesoran para, a partir de procesos dialógicos y contextuales, dar lugar a espacios de aprendizaje en red. En este sentido, comprendemos cómo las prácticas artísticas colaborativas ponen en juego una pedagogía de carácter colectivo y rizomático, en tanto que el conocimiento en ellas es continuamente mediado y dinamizado entre nodos (agentes). Es decir, las prácticas colaborativas generan y disponen un conocimiento que es de carácter relacional, fluye y se media continuamente, y su potencia deriva precisamente de la capacidad por hacer que sea dispersado, multiplicado, reapropiado, continua y abiertamente. De las relaciones y conexiones múltiples que se dan en la 
colaboración, podemos extraer una de las cualidades elementales y diferenciales de las prácticas que analizamos en este trabajo: su condición transfronteriza.

En el estudio de las políticas relacionales que emergen y se emulsionan en las redes de trabajo colaborativo, que nos hemos propuesto señalar en este artículo, se construyen también unos modelos de política cultural que nos interesa problematizar y proponer como oportunidad para entender otros modos posibles de práctica instituyente. Nos referimos, fundamentalmente, a las aportaciones que pueden hacer las experiencias de trabajo en comunidad o con públicos específicos para pensar una nueva institucionalidad cultural, para desarrollar espacios de producción y debate en los que sea posible una organicidad de carácter más horizontal o democrática. Es decir, espacios o proyectos instituyentes que fueran permeables a formas de organización experimentadas en otros ámbitos: por ejemplo, en el tercer sector, donde las fórmulas asamblearias o de comisiones ciudadanas acumulan una larga historia de ensayos, o en el sector científico-tecnológico, donde son comunes los modelos de trabajo basados en estructuras moleculares hiperconectadas o en red. La apuesta de este trabajo se dirige a indagar fórmulas y referencias que pudieran aportarnos vías por las que aprender y experimentar mecanismos con los saber conjugar las fricciones, disensos y antagonismos que caracterizan los espacios plenamente democráticos, para con ello imaginar y diseñar una espacialidad potencialmente híbrida, mutable, en movimiento, ... proyectos y experiencias instituyentes donde las políticas culturales que se pudieran promover dieran un paso más allá de los programas de representación, comunicación y acceso, y permitieran la generación de políticas de participación y agencia.

Cuando proponemos la categoría o denominación "laboratorios artísticos colaborativos" para referirnos a la espacialidad crítica que parece emerger en los cruces de las redes de colaboración, queremos remitir a la posibilidad de vislumbrar modelos de trabajo contra-hegemónicos con los modos neo-liberales imperantes. Frente a la cultura mercantilizada y proclive a la espectacularización que pueden ejemplificar los grandes centros artísticos -modelo Guggenheim- asociados al turismo cultural trans-nacional, surgen en nuestras ciudades territorios que tratan de instituirse en polos de tensión y alternativa crítica a los discursos de dominación. Se trata de espacios en muchos casos frágiles, en cuanto adolecen de una estructura soldada o fija que los haga inquebrantables, y difusos, por lo complejo de dibujar un perfil homogéneo al que poder circunscribirlos, pero en estas características, en las que podrían encontrar su debilidad, se fundamenta también su fortaleza. Su capacidad para improvisarse, adaptarse y rearticularse permanentemente, les otorgan una cualidad resbaladiza que dificultan su capción. Su sostenibilidad no depende de fijar una apariencia o contorno formal invariable, sino de estar diseñadas mediante estructuras flexibles y reconfigurables, capaces de mutar y adaptarse ecológicamente. Su posición esta en el "entre", en la franja o límite en la que poder configurarse como bisagra o lugar entremedias (BHABHA, 2002), es decir, como espacios híbridos que surgen dentro del sistema, pero se posicionan como resistencia a él.

Esta cualidad "entremedias" o "intersticial" hace que estos espacios de experimentación colaborativa se constituyan en territorios de traducción y subversión tanto de los discursos dominantes como de los del "otro" supuestamente oprimido. 
Es decir, hablamos de un espacio híbrido que se sitúa en la inestable frontera del estar continuamente entre el dentro y el afuera. Ahí radica su capacidad de mantener cierta posibilidad de autonomía en su posición: en permitir y saberse mover y estacionar voluntariamente en puntos distintos de este eje de tensión. La naturaleza crítica de esta espacialidad deviene de su constante articulación entre posiciones diversas, y con ello, de su capacidad de mediar y traducir los signos y discursos dados, generándose a partir de esta movilidad la posibilidad de efectuar rupturas de las relaciones de dominio y sumisión que pudieran ser identificadas. Esto nos lleva a pensar en la conformación de espacios y modelos de funcionamiento orgánico donde la subversión de posiciones sea rearticuladora, no sólo discursivamente, sino también respecto a los modos de vinculación y participación de los agentes colaboradores en ellos. De ahí que el trabajo político esencial que notamos emerger en las experiencias instituyentes, que hemos tratado de presentar y analizar críticamente, sea el de deconstruir y transformar los mecanismos y dispositivos que imponen y clausuran unos modos de relación y participación predeterminados e inamovibles. En cambio, el estudio desarrollado en esta investigación ha permitido entender otros marcos de trabajo que, sin obviar sus complejidades y controversias, presentan otros modos relacionales posibles, laboratorios experimentales donde se articulan formas de participación basadas en el intercambio y desborde continuo de los posicionamientos de los agentes implicados.

De este modo, pensamos en estos laboratorios artísticos colaborativos como plataformas de producción y circulación de conocimientos donde se dan transvases a diversos niveles: de los roles y posiciones de los sujetos y organismos involucrados, transgrediendo el estatus tradicional que otorga unas condiciones fijas a productores y públicos; de las condiciones y estructuras de organización, subvirtiendo los modelos jerárquicos verticales por la experimentación con modos de trabajo y colaboración en red; de la propia naturaleza y dimensión de estos espacios, al combinar en ellos tareas de investigación, producción cultural, comunicación y difusión, educación, desde una perspectiva abierta e inclusiva y con marcado carácter social, es decir, vinculando y generando estrategias de participación en las que se vean comprometidos sujetos y organismos dispares. En este aspecto último concluye su aportación más decisiva: en indagar y proponer fórmulas, que son también retos, para repensar las políticas culturales oficiales, y con ello también para cuestionar las prácticas asentadas de los distintos agentes (artistas, comisarios, investigadores, críticos, etc.) y las propias instituciones que articulan tradicionalmente el campo artístico. La condición intermedia e híbrida de estos espacios permite armar una crítica más atenta a las convenciones y normas del contexto cultural y sus herencias asentadas. Esto posibilita comprender el bagaje acumulado sin comprometer la crítica productiva que pudiera permitir imaginar y experimentar modelos de funcionamiento que, aún expuestos al riesgo de lo inédito, avanzaran cualidades atisbadas en otros ámbitos sociales y culturales. Aquí el reto es mayúsculo y la atención debería ser extrema -ya que no cabe una fórmula ni una estrategia definitiva o conclusiva- para intentar atisbar el devenir de los modos de vida sociales e intentar acompasar y aprender de las nuevas y continuas experiencias de organización/acción que acontecen. 


\section{Referencias}

Barandiaran, X. (2006). Hacklabs, Hacksmeetings. En VV. AA. Ciberactivismo. Sobre usos políticos y sociales de la red (pp. 139-155). Barcelona: VIRUS.

Bhabha, H. (2002). El lugar de la cultura. Buenos Aires: Manantial.

Boltanski, L. y Chiapello, È. (2002). El nuevo espíritu del capitalismo. Madrid: Akal.

Callon M., Lascoumes P. y Barthe Y. (2009). Acting in an Uncertain World. An Essay on Technical Democracy. Cambridge: The MIT Press, coll. "Inside Technology".

Cobo, C. y Pardo, H. (2007). Planeta Web 2.0. Inteligencia colectiva o medios fast food. Barcelona / México DF: Grup de Recerca d'Interaccions Digitals, Universitat de Vic. Flacso México.

Collados, Antonio y Rodrigo, Javier. (2012). TRANSDUCTORES: Pedagogías en red y prácticas instituyentes. Granada: Centro de Arte José Guerrero.

Deleuze, G. y Guattari, F. (1985). El Anti-Edipo. Capitalismo y esquizofrenia. Barcelona: Paidós.

Garcés, M. (2010) Dar que pensar. Extraído el 5 de abril, 2013 de http://www. espaienblanc.net/IMG/pdf/Dar_que_pensar.pdf

Giroux, H. A. (1997). Cruzando límites. Trabajadores culturales y políticas educativas. Barcelona: Paidós.

Gual, J; Salvini, F. (2010). Be Network, my friend. Extraído el 5 de abril, 2013 de http://radical.temp.si/2010/03/be-network-my-friend-by-joan-miquel-gual-andfrancesco-salvini-members-of-universidad-nomada/

Holmes, Brian. Makrolab, or the art of transition. Extraído el 24 de enero, 2013 de http://brianholmes.wordpress.com/2007/03/27/coded-utopia/

Hyde Park Art Center (2007). Pedagogical Factory: Exploring strategies for an educated city. Extraído el 5 de abril, 2013 de http://www.hydeparkart.org/ exhibitions/the-pedagogy-project

Laddaga, R. (2010). Estética de laboratorio. Buenos Aires: Adriana Hidalgo.

Lafuente, A. (2008). Laboratorio sin muros. Inteligencia colectiva y comunidades de afectados. Extraído el 5 de abril, 2013 de http://digital.csic.es/handle/10261/2899

Latour, B. (1992). Ciencia en acción. Cómo seguir a los científicos e ingenieros a través de la sociedad. Barcelona: Ed. Labor.

Machal-Cajigas, A. (2004). El colaboratorio: Un nuevo enfoque hacia la investigación científica. Extraído el 5 de abril, 2013 de http://mit.ocw.universia.net/STS.035/NR/ rdonlyres/Science--Technology--and-Society/STS-035Spring2004/61 A388622CA3-447D-ACC3-3FC482DF93CB/0/11antoinne.pdf

Makrolab (1999). Vision. Extraído el 3 de diciembre, 2013 de http://makrolab. ljudmila.org/

Möntmann, N. (2011). Operar con redes organizadas. CARTA, 2, 95-96

Mouffe, C. (2007). Prácticas artísticas y democracia agonística. Barcelona: MACBA-UAB.

Nowotny, H. (2006). The potential of the transdisciplinarity. Extraído el 5 de abril, $2013 \mathrm{http}: / /$ helga-nowotny.eu/downloads/helga_nowotny_b59.pdf

Pérez de Lama, José, de Soto, Pablo y Moreno, Sergio. (2003). FADAIAT, por los espacios de la frontera suroeste de la Europa Fortaleza. a-mínima, 9, 72-79. 
Petcou, C.; Petrescu, D.; Querrien, A. (2007). What Makes A Biopolitical Place? A Discussion with Toni Negri. En VV.AA. URBAN/ACT a handbook for alternative practice (pp. 290-299). París: AAA/PEPRAV.

Petcou, C;. Petrescu, D. (2007). Acting Space: transversal notes, on-the-ground observations, and concrete questions for us all. En VV.AA. URBAN/ACT a handbook for alternative practice (pp. 319-328). París: AAA/PEPRAV.

Petrescu, D. (2005). Losing control, keeping desire. En Blundell jones et alt. Architecture \& Participation (pp. 43-64). Londres and Nueva York: Taylor \& Francis Group.

Remesar, Antoni. (2005). Arte contra el pueblo: los retos del arte público en el s.XXI Extraído el 10 de febrero, 2013 de http://ub.academia.edu/AntoniRemesar/ Papers/443843/Arte_contra_el_pueblo_los_retos_del_arte_publico_en_el_s.XXI

Ribalta, J. (2009). Experimentos para una nueva institucionalidad. Extraído el 15 de febrero, $2013 \mathrm{de} \mathrm{http://marceloexposito.net/materialesteoricos/1969.html}$

Rodrigo, J. (2007). Educación artística y prácticas artístico-colaborativas: territorios de cruce transversales [en línea]. En: Coca, P. y Montero, P (Eds.). Arte contemporáneo y educación: un diálogo abierto. Valladolid: Junta de Castilla y León, Consejería de Cultura y Turismo.

Rossiter, Ned. (2011). Can Organized Networks Make Money for Designers?. Cit. pos. Möntmann, Nina. Operar con redes organizadas. CARTA, 2, 95-96

Sansonetti, L. (2008). Centros sociales de segunda generación. VV.AA. Autonomía y Metrópolis: Del movimiento okupa a los centros sociales de segunda generación (pp. 13-19). Málaga: Centro de Ediciones de la Diputación Provincial de MálagaCedma.

The Public School (2012). About. Extraído el 5 de abril, 2013 de http:// la.thepublicschool.org.

Simonson, L. (2009). Entrevista a Sean Dockray, miembro de Telic Arts Exchange. Extraído el 10 de febrero, 2013 de http://blog.art21.org/2009/11/12/looking-atlos-angeles-the-public-school/

Stoyckard Institute (2007). Pedagogical Factory. Extraído el 10 de febrero, 2013 de http://www.stockyardinstitute.org/pedagogical-factory-2007/

Universidad Nómada. (1998). Prototipos mentales e instituciones monstruo. Algunas notas a modo de introducción. Extraído el 23 de noviembre, 2013 de http://eipcp. net/transversal/0508/universidadnomada/es

VV.AA. (2005). Informe mundial: Hacia las sociedades del conocimiento. Extraído el 5 de abril, 2013 de http://www.unesco.org/es/worldreport

VV.AA. (2006). Fadaiat. Libertad de movimiento + libertad de conocimiento. Málaga: Imagraf impresores. 